\title{
Estudios bibliométricos de la influencia tecnológica en el desarrollo productivo sostenible en artículos publicados en SCOPUS 2012 - 2016
}

\author{
Bibliometric studies of the technological influence in the sustainable \\ productive development in articles published in SCOPUS 2012 - 2016
}

Jonathan Soto Hernández

Carlos Villamar Monsarrate

Jorge Luis Vinueza Martínez

Alexandra Astudillo Cobos

Mirella Correa Peralta

Universidad Estatal de Milagro, Ecuador

Autor corresponsal: jsotoh1@unemi.edu.ec, cvillamarc@unemi.edu.ec, jvinuezam@gmail.com, aastudilloc@unemi.edu.ec, mcorreap@unemi.edu.ec

Fecha de recepción: 16 de Octubre de 2017 - Fecha de aceptación: 15 de Abril de 2018

Resumen: Este trabajo fue un estudio documental de la identidad de la tecnología en el desarrollo productivo sostenible de los gremios artesanales en la base de datos SCOPUS, abordando desde el punto de vista de las variables: desarrollo productivo y tecnología como un análisis general de los estudios realizados por varios autores. La metodología fue 2 consultas relacionadas al desarrollo productivo TITLE-ABS-KEY ( desarrollo AND productivo ) y productive AND development AND craft AND guild AND ( LIMIT-TO ( PUBYEAR, 2016 ) OR LIMIT-TO ( PUBYEAR , 2015 ) OR LIMIT-TO ( PUBYEAR, 2014 ) OR LIMIT-TO ( PUBYEAR , 2013 ) OR LIMIT-TO ( PUBYEAR, 2012 )) AND ( LIMIT-TO ( DOCTYPE, "ar" )) en 2 fases: 1. Obtener información. 2. Analizar y evaluar información validando autores, artículos y fuentes obtenidas. Este estudioo cuantitativo permitió concluir la asociación de 10 autores del artículo Replacing soybean for winter peas in heavy-pig diets: Productive impact of the level of protease inhibitors en la revista ITEA Informacion Tecnica Economica Agraria, revista del cuartil Q3; en el caso de las revistas Cadernos de Saude Publica de Brasil cuenta con el 35\% de participación Latinoamericana, y el artículo Precocious albion: A new interpretation of the British Industrial revolution, publicada en Annual Review of Economics del 2014 resalta la influencia del desarrollo productivo, importancia del capital humano y calidad del trabajo asociadas con el alto nivel de nutrición, mejores instituciones y el alto nivel de aprendizaje.

Palabras Claves: estudio bibliométrico; tecnología; base de datos; producción; publicaciones

Abstract: This work was a documentary study of the identity of the technology in the sustainable
productive development of the artisan guilds in the database SCOPUS, approaching from the point
of view of the variables: productive development and technology as a general analysis of the
studies carried out by several authors. The methodology was 2 queries related to the productive
development TITLE-ABS-KEY (productive AND productive) and productive and Development
and Craft and Guild and (Limit-To (Pubyear, 2016) Or Limit-To (Pubyear, 2015) Or Limit-To 
(Pubyear, 2014) Or Limit-To (Pubyear, 2013) Or Limit-To (Pubyear, 2012)) And (Limit-To (Doctype, "ar")) in 2 phases: 1. Get information. 2. Analyze and evaluate information validating authors, articles and sources obtained. This quantitative study allowed to conclude the association of 10 authors of the article Replacing soybean for winter peas in heavy-pig diets: Productive impact of the level of protease inhibitors in the ITEA magazine Technical Information Economica Agraria, magazine of the quartile Q3; in the case of Brazil's Cadernos de Saude Publica, it has 35\% Latin American participation, and the article Precocious albion: A new interpretation of the British Industrial Revolution published in the Annual Review of Economics of 2014 highlights the influence of productive development, the importance of human capital and the quality of work associated with the high level of nutrition, better institutions and the high level of learning.

Key words: bibliometric study; technology; database; production; publications

\section{Introducción}

La temática: Identidad de la tecnología en el desarrollo productivo sostenible de los gremios artesanales, analizada desde el punto de vista de las ciencias organizacionales, tecnológicas, abarca el estudio documental obtenido en la base de datos de SCOPUS, las variables: desarrollo productivo y tecnología definida en el tópico general, son estudiadas con la revisión bibliográfica relacionada a desarrollo productivo e influencia tecnológica, de manera cómo el crecimiento y la tecnología influye en el contexto de globalización mundial en los países, como lo afirma Pérez C (2010) acerca de la revolución industrial, revolución tecnológica o el impacto de la innovación crean una potencial riqueza social, trayendo consigo con la cadena de valor, mercados globales y las competencias tecnológicas la diversificación de oportunidades a los países; es así, como la hipersegmentación de mercados por la alta rentabilidad o competencia de los precios. Pero a todo esto las tecnologías de la información y la comunicación (TICs) innovan los medios de trabajos.

Uno de los casos es el campo de la logística que en tareas eficaces en la administración y resultados de la cadena de suministros, pues la formación, eficiencia y sostenibilidad se pueden obtener con el uso intensivo de las TIC'S, circunstancia que se produce con la estandarización de las tecnologías aplicadas a la gestión logística. Con la inclusión de sitios web o el comercio electrónico permite identificar ampliar la cartera de proveedores. En el caso de los sectores artesanales el manejo de las redes sociales es mínimo y los empresarios aun no tienen claros los beneficios que contribuyen.

En el Ecuador el gobierno impulsa el desarrollo innovador y tecnológico como una fuente inagotable de recursos; de ahí el Ministerio Coordinador de Conocimiento y Talento Humano tienen como objetivo fortalecer la Sociedad del Conocimiento, involucrando a la Secretaría de Educación Superior, Ciencia, Tecnología e Innovación (Senescyt), el Servicio Nacional de Contratación Pública (SERCOP) instituciones que apoyan con participaciones a través de concursos, conferencias u otros, con el objetivo de incentivar al sector privado a ser parte del impulso tecnológico y productivo del país; como es producir: teléfonos con tecnología IP, transformadores de alto voltaje, camas eléctricas, lámparas de fototerapia, multímetros, relojes biométricos, partes automotrices, entre otros productos; para así reducir importaciones de forma que favorezca la balanza comercial del país. 
Además, en este estudio se analiza variables bibliométricas, en este sentido se toma como referencia a Guerra et.al (2013) quien hace un análisis de la gestión de proyectos de innovación a partir de los registros documentales en la base de datos de SCOPUS, señalando técnicas de administración y planificación, a fin de profundizar en conocimiento científico alrededor de desarrollo productivo y tecnológico lo que implica identificar estudios previos, con enfoque de recolectar estudios publicados de forma cuantitativa para genera un análisis y conclusiones. Para este trabajo se obtuvo 2 grupos de fuentes de información en SCOPUS para limitar el alcance de los resultados y evaluar indicadores de autores, revistas, año, países, instituciones entre otros, y con la finalidad de conocer estudios previos de otros países, en función del desarrollo productivo y la tecnología.

\section{Marco teórico}

La Teoría general de los sistemas desde la complejidad en el campo del conocimiento sistémico, comprende una tendencia e integración de sus partes como lo menciona Von Bertalanffy (1993); basado en esta teoría desde de fundamento, se ha de: identificar conceptos o leyes, identificar el desarrollo de modelos teóricos para minimizar repetición de esfuerzo teórico, y promover la unidad de ciencia especializada como enfoque de los sistemas.

En el caso del desarrollo productivo, se ha de identificar varios estudios como el de Osta (2007), en su estudio de redes empresariales y relaciones para el desarrollo productivo destaca el papel de los proyectos emprendedores incluyendo la innovación, cooperación, coordinación, intercambio entre todos los sectores, analiza un sector como es el caso de las franquicias que han permitido la generación de empleo e incentivando las actividades productivas formales. Además Pérez C (2010), en su estudio denominado el trabajo de dinamismo tecnológico e inclusión social en América Latina: una estrategia basada en los recursos naturales, destaca la inclusión y revolución de la tecnología como parte de nuevas posibilidades en el mercado, posicionamiento de redes globales y opciones tecnológicas. Ambos autores señalan términos como: innovación, intercambio-redes, producción emprendimiento como aporte económico a la sociedad.

Además, Saubidet et al. (2012), aplicó un estudio de experimentación de la educación en el procesamiento pesquero, el objetivo principal era comparar esquemas de evaluación de la viabilidad tecnológico como herramienta productiva y como elemento importante en el proceso de aprendizaje. También, Gedajlovic et al. (2012), señalan a través de una investigación en empresas familiares el aporte a revistas de gestión, emprendimiento y finanzas, resaltando en gran medida aspectos de integración y organización. Así como lo manifiesta Burt and Atkinson (2012), la creatividad puede ser un camino importante que resalta el bienestar de quienes aportan en actividades de producción. Es así, como de las universidades, sector productivo y sociedad deben tener una conexión directa basada en la identificación de necesidades para crear empleo y diversificación de productos generando una competencia de mercados.

Ogilvie and Carus (2014), hace un análisis de las instituciones en relación al crecimiento económico y otros componentes sistémicos lo que genera la integración de sus partes. También Desmet and Parente (2014), analiza la teoría de relación de U invertida entre gremios y el tamaño del mercado, pues en función de mercados pequeños beneficios insuficientes para cubrir la inclusión de nueva tecnología. Kelly, Mokyr, and Gráda (2014) hace hincapié el desarrollo en 
aspectos de producción en Gran Bretaña desde el siglo XVIII, generando mejor calidad de vida que genera mejor aspectos de nutrición, obteniendo mejores instituciones y mejor educación.

Gil, White, and Caceres (1990), describe el aporte financiero en el desarrollo productivo en Chile específicamente en biotecnología como son becas, proyectos productivos vinculando el sector productivo y la academia. Además Viana et al. (2016), manifiesta que en el desarrollo de las sociedades influye el desarrollo tecnológico, pues los estados están involucrados en incluir estrategias y decisiones tecnológicas como el caso de Brasil a través de políticas de salud que incentivan el mejorar la calidad de vida de la población. Además Jacometti et al. (2016), en su estudio de medir el impacto de las políticas públicas y su impacto en el desarrollo económico resaltando el impacto de las relaciones sociales y la competencia en los pequeños grupos de las empresas.

Oleas-Montalvo (2016), se cuestiona sobre la economía abierta en un país, señalando un ejemplo en el Ecuador destacando el desarrollo de la industria básica, sustitución de las importaciones, diversificación de la oferta con el objetivo de crear una sociedad basada en la producción de servicios y menos dependiente de recursos, para generar mejor capacidad en ciencia, tecnología e innovación.

Como lo menciona Guerra et.al(2013), el profundizar el comportamiento de los proyectos de innovación y las herramientas a utilizar, es fundamental potenciar el desarrollo para profundizar los conceptos que estén relacionados a este trabajo, y destaca en la conclusión la evolución en función a producción científica, analizando otras bases de datos como son Scielo, Redalyc, Latindex u otros.

Para Vargas-Quesada et.al (2010), en la publicación de estructura de colaboración científica española en bibliometría y documentación integran aspectos importantes de variables identificando 21 componentes donde identifica la colaboración de información científica con afiliación de España, de este estudio se puede inferir la importancia de la validación de información a fin de cumplir criterios homogéneos para la visualización o representación gráfica, donde analiza la correlación entre producción y la media central generando una evaluación cuantitativa para de ahí conformar una subred; de ahí concluye que la metodología evalúa principalmente la colaboración y participación de autores en cuanto a producción científica. De esta forma este trabajo se desarrolla con la finalidad de identificar a SCOPUS no solo como medio de búsqueda de información sino como fuente de análisis de datos, que permita identificar variables y evaluar con otro medio como es SJR, generando una validación del cuál es la fuente idónea al consultar datos relevantes.

Los autores refieren a incluir en el desarrollo productivo de un país basado en las políticas del estado orientado a la necesidad del estado, generando guías en aspectos de emprendimiento con la inclusión de tecnología que permita contribuir el impacto social y económico integral, que en cierta forma fortalece la I+D+I a través de la vinculación los sectores productivos y las instituciones de educación superior, generan su contribución responsable y la obtención de recursos y servicios eficientes. 
Granda-Orive, et. al. (2013), En su estudio de ciertas ventajas de Scopus sobre Web of Science en un análisis bibliométrico sobre tabaquismo, inicia como empezó la Web of Science (WoS), para que apareció la Journal Citation Report (JCR); en comparación con SCOPUS ha facilitado este su navegación, presenta el factor de impacto, tomando información desde SCImago Journal Rank (SJR); no evidencia cual es mejor o no, sino la importancia de ambas bases de datos donde son complementarias entre ambas. A esto el autor presenta sus etapas de recogida de datos, homogeneidad de información, indicadores a analizar, estadística, presentación de resultados; una de sus conclusiones sugiere el justificar el por qué seleccionar una base de datos como fuente de información y el método a utilizar. La conexión y participación de los estudiantes dentro de comunidades u organizaciones, hacen que la colaboración entre equipos sea más efectiva, participativa y sostenible a la hora de gestionar servicios y procesos para la organización, conllevando a estos ciertos mecanismos que faciliten al análisis de resultados, ya sean estos internos o externos. Dichos resultados les permitirán a los estudiantes de grupos o comunidad mejorar capacidades intelectuales manteniendo así una mejora en cuanto a participación y comunicación en el desarrollo y análisis de cierto software o aplicativos. En ciertos casos se ha podido constatar que la tecnología es un fenómeno esencial en cuanto al manejo y manipulación de la información, ya que ha transformado un ambiente competitivo de trabajo, facilitando mayor flexibilidad en procesos dentro de la organización. Los nuevos mecanismos de trabajos ayudan relativamente al desarrollo eficiente y eficaz de nuevos argumentos, con bases para las directrices de nuevas herramientas que ayuden a la comunicación entre los empleados en una organización, promoviendo así ideas diferentes pero innovadoras. Por lo tanto, los procesos que conllevan son enfocados en investigaciones, evaluando herramientas que ayuden a la implementación de procesos y desempeño de la organización.

Para el Ecuador a través del Ministerio Coordinador de Producción, empleo y competitividad destaca en sus indicadores la proyección de crecimiento de acuerdo a la Comisión Económica para América Latina y el Caribe (CEPAL) como factor de proyección de crecimiento al 2015 en $0,4 \%$ con la tasa de desempleo nacional a junio del 2016 de $5.32 \%$ y una tasa de ocupación adecuada al 2016 en 41,05\%; sin embargo el crecimiento interanual del sector de la manufactura se encuentra a 2016 en $-1,3 \%$ y para el sector de la agricultura en 2.6\%; indicadores que están en función al Plan Nacional del Buen Vivir PNBV 2013-2017 de incrementar la producción para generar ingresos económicos; a más de esto en el marco de la tecnología, innovación y crecimiento menciona este desarrollo debe estar enfocado en el bioconocimiento y producir bienes o servicios que satisfagan las necesidades del país, aportando a la matriz productiva, es decir, la inversión en el conocimiento a investigación, desarrollo e innovación $(\mathrm{I}+\mathrm{D}+\mathrm{I})$. De ahí, como la producción en conocimiento generan nuevas oportunidades económicas a través de proyectos innovadores, donde la cultura de investigación permite generar un fortalecimiento en la educación, en los sectores productivos y comerciales, como lo presenta la Secretaría Nacional de Planificación y Desarrollo (SENPLADES), el indicador de producción en el Ecuador según SCImago está por debajo de los países de Latinoamérica como Chile y Colombia; siendo el objetivo 10 del PNBV de Transformar la matriz productiva obtenido al 2012 un $20 \%$ está distribuido en bienes primarios y basado en recursos, por lo tanto estrategias como articular la educación con la investigación que fomente en las industrias la pertinencia tecnológica y de producción de manera sustentable articulado a las necesidades de la sociedad.

\section{Metodología}


Para Darosi et. al (2014) el análisis de estudio documental comprende medir, identificar y analizar la documentación bibliográfica; entonces, este trabajo consistió en evaluar bibliográficamente publicaciones de la base de datos de Scopus relacionado al tema de "desarrollo productivo".

Obteniéndose 14 documentos (Tabla 1), consultando:

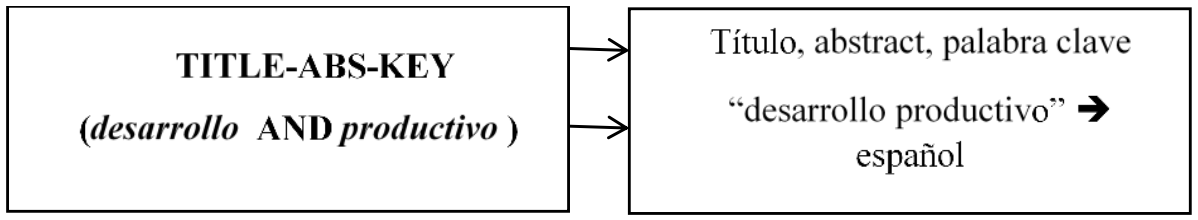

Y, 9 documentos (Tabla 2), en relación a la búsqueda

\begin{tabular}{|c|c|}
\hline $\begin{array}{l}\text { Productive AND development } \\
\text { AND craft AND guild } \\
\text { AND ( LIMIT- } \\
\text { TO (PUBYEAR, 2016) OR LIMIT- } \\
\text { TO (PUBYEAR, 2015) OR LIMIT- } \\
\text { TO ( PUBYEAR, 2014) OR LIMIT- } \\
\text { TO ( PUBYEAR, 2013) OR LIMIT- } \\
\text { TO ( PUBYEAR, 2012)) AND } \\
\text { (LIMIT-TO ( DOCTYPE, "ar")) }\end{array}$ & $\begin{array}{l}\text { Desarrollo productivo } \\
\text { Gremio de artesanos } \\
\text { Del } \\
2016 \\
2015 \\
2014 \\
2013 \\
2012 \\
\text { Artículos }\end{array}$ \\
\hline
\end{tabular}

La metodología fue búsqueda bibliográfica y análisis de la estructura de información de la base de datos de SCOPUS, para ello se definió dos fases generales: Primero, obtención de información delimitando las variables a consultar, Segundo, evaluación de información para análisis de resultados cuantitativos y cualitativos, como se observa en la Figura 1.

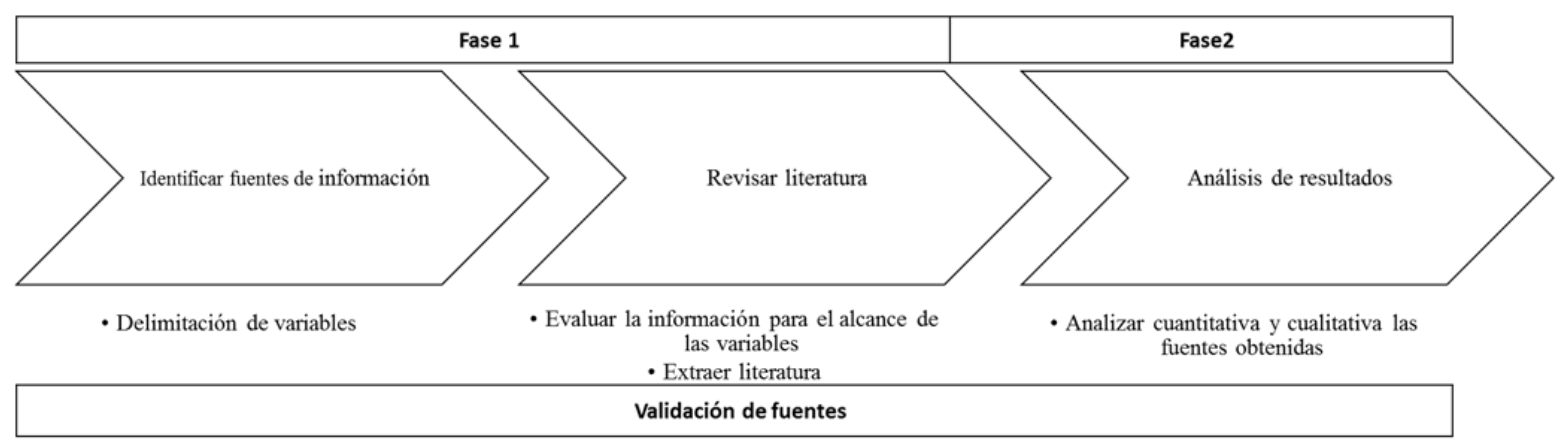

Figura 1. Obtención de la información Fuente: Elaboración propia

Tabla 1. Publicaciones de sector productivo en la base de datos de SCOPUS

\begin{tabular}{lll}
\hline Año & Revista & Artículos \\
\hline
\end{tabular}




\begin{tabular}{|c|c|c|}
\hline 1981 & $\begin{array}{l}\text { "Revista de } \\
\text { Logopedia, Foniatria } \\
\text { y Audiologia" }\end{array}$ & Logopedia, integración y deficientes auditivos(Bruno Buqueras, 1981) \\
\hline 1985 & $\begin{array}{l}\text { Journal of Animal } \\
\text { Breeding and } \\
\text { Genetics }\end{array}$ & $\begin{array}{l}\text { Untersuchungen über die Nutzleistung des Djallonkéschafes: Ein Beitag zur } \\
\text { Kenntnis einer westafrikanischen Schafrasse(Filius, Weniger, \& Teuscher, 1985) }\end{array}$ \\
\hline 1990 & $\begin{array}{l}\text { Archivos de } \\
\text { Biologia y Medicina } \\
\text { Experimentales }\end{array}$ & $\begin{array}{l}\text { Financing research in Chile in biotechnology and other areas related to the } \\
\text { productive sector [Financiamiento de la investigación en Chile en biotecnología y } \\
\text { en otras áreas relacionadas con el sector productivo.](Gil, White, \& Caceres, } \\
\text { 1990) }\end{array}$ \\
\hline 2007 & Espacios & $\begin{array}{l}\text { Franchises: Enterprise networks and relations for the productive development } \\
\text { [Franquicias: Redes y relaciones empresariales para el desarrollo } \\
\text { productivo](Osta, 2007) }\end{array}$ \\
\hline 2008 & $\begin{array}{l}\text { Boletin de la } \\
\text { Asociacion de } \\
\text { Geografos Espanoles }\end{array}$ & $\begin{array}{l}\text { Tourism, outdoor recreation and public use in natural parks. A proposal for the } \\
\text { conservation of the environmental values and the local economic development } \\
\text { [Turismo, actividades recreativas y uso público en los parques naturales. } \\
\text { Propuesta para la conservación de los valores ambientales y el desarrollo } \\
\text { productivo local](Clavé, Boqué, \& Salvat, 2008) }\end{array}$ \\
\hline 2009 & $\begin{array}{l}\text { Archivos de } \\
\text { Zootecnia }\end{array}$ & $\begin{array}{l}\text { Parda de Montaña replacement heifers: Nutrition and productive performance } \\
\text { [Recría de terneras parda de montaña: Alimentación y desarrollo } \\
\text { productivo](Bodas, López-Campos, \& Mantecón, 2009) }\end{array}$ \\
\hline & $\begin{array}{l}\text { Revista de la } \\
\text { Facultad de Ciencias } \\
\text { Agrarias }\end{array}$ & $\begin{array}{l}\text { Influence of light interception on vegetative and productive development of } \\
\text { peaches cv. Elegant Lady [Influencia de la intercepción lumínica en el desarrollo } \\
\text { vegetativo y productivo de durazneros cv. elegant lady](Dussi et al., 2009) }\end{array}$ \\
\hline
\end{tabular}

2010 Journal of Technology Management and Innovation

2012 Revista Electronica de Veterinaria

Model management development plan local production: The case of Nova Friburgo, Brazil [Modelo de gestión de un plan de desarrollo productivo local: El caso de Nova Friburgo, Brasil](Moré, Lima, \& de Almeida, 2010)

Productive, didactic module for the development of outdoor learning practices in the career of Technician in Aquaculture and Fisheries Processing [Módulo didáctico-productivo para el desarrollo de prácticas de aprendizaje extráulicas, en la carrera Tecnicatura en Acuicultura y Procesamiento Pesquero](Saubidet, Pérsico, Lucero, \& Imeroni, 2012)

2013 Revista de Investigaciones Veterinarias del Peru

Development and validation of a model to predict the optimum requirement of essential amino acids and performance in commercial laying hens [Desarrollo y validación de un modelo de predicción del requerimiento óptimo de aminoácidos esenciales y del comportamiento productivo en ponedoras comerciales](Salvador \& Guevara, 2013)

2016 Cadernos de Saude Publica

Development policy for the Brazilian health industry and qualification of national public laboratories [A política de desenvolvimento produtivo da saúde e a capacitação dos laboratórios públicos nacionais] [La política de desarrollo productivo en salud en Brasil y la cualificación de los laboratorios públicos nacionales](Viana, da Silva, Ibañez, \& Iozzi, 2016)

Latin American Perspectives Revista de Citizens' revolution and international integration(Oleas-Montalvo, 2016)

Administracao

An analysis of the effectiveness of public policies relating to local productive arrangements (LPA'S) on local development from the perspective of institutional theory [Análise de efetividade das políticas públicas de arranjo produtivo local para o desenvolvimento local a partir da teoria institucional] [Análisis de la efectividad de las políticas públicas de arreglo productivo local para el desarrollo local desde la teoría institucional](Jacometti, Castro, Gonçalves, \& Costa, 2016) 


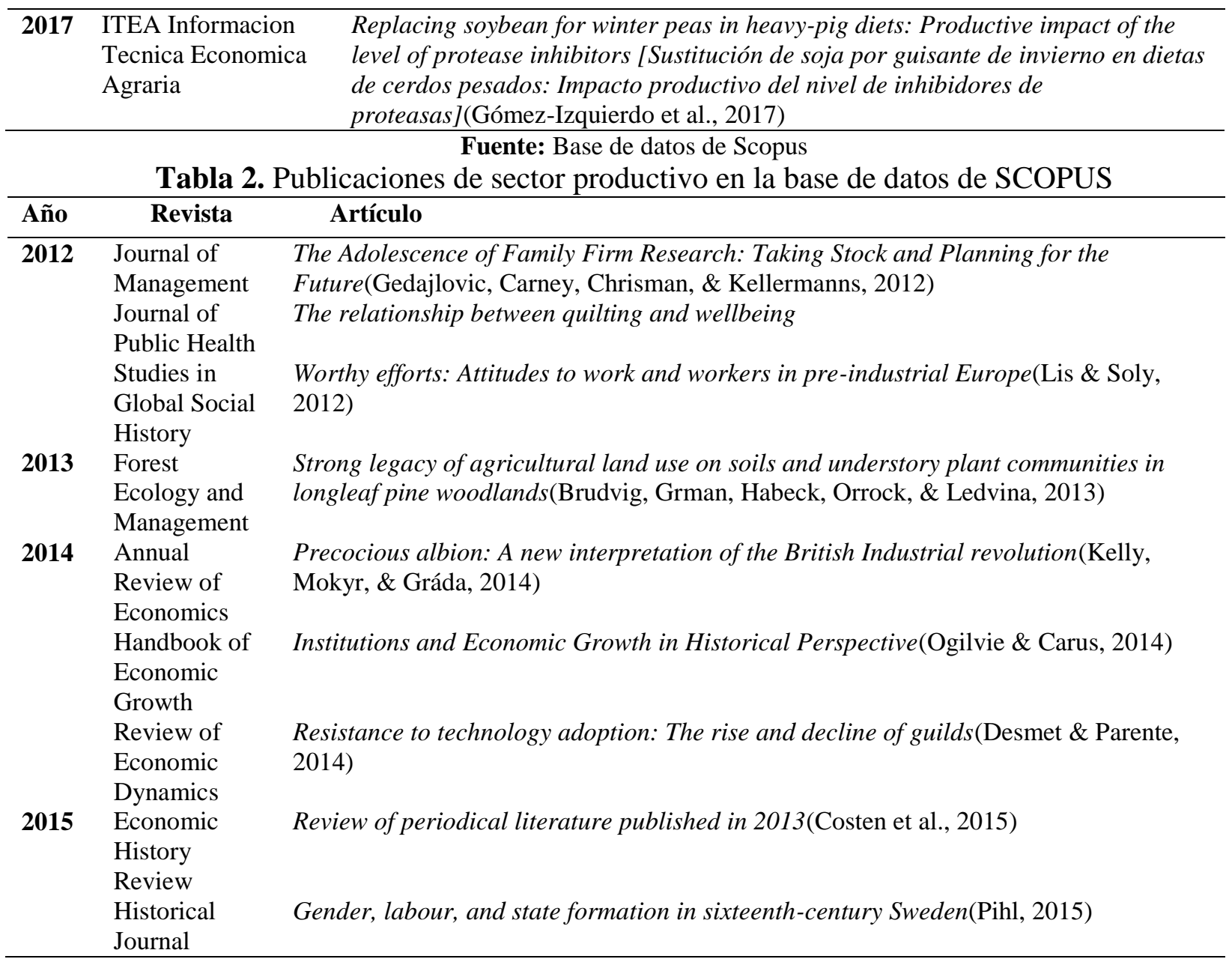

Fuente: Base de datos de Scopus

\section{Resultados - "Desarrollo productivo"}

$\mathrm{El}$ artículo Replacing soybean for winter peas in heavy-pig diets: Productive impact of the level of protease inhibitors [Sustitución de soja por guisante de invierno en dietas de cerdos pesados: Impacto productivo del nivel de inhibidores de proteasas], tiene la participación de 10 autores en el 2017(Tabla 3).

Tabla 3. Número de artículos publicados en "Methodology RUP" por participación de autores en la base de datos Scopus del 2014 al 2017

\begin{tabular}{lcccccc}
\hline Año & Publicaciones & Autores & & & \\
\hline & & Simple & Doble & Triple & Cuádruple & Séxtuple Décuple \\
1981 & 1 & 1 & & & & \\
1985 & 1 & & 1 & \\
1990 & 1 & & 1 & \\
2007 & 1 & 1 & 1 & \\
2008 & 1 & & 1 & 1 \\
2009 & 2 & & & & \\
\hline
\end{tabular}




\begin{tabular}{|c|c|c|c|c|c|c|c|}
\hline 2010 & 1 & & & 1 & & & \\
\hline 2012 & 1 & & & & 1 & & \\
\hline 2013 & 1 & & 1 & & & & \\
\hline 2016 & 3 & 1 & & & 2 & & \\
\hline 2017 & 1 & & & & & & 1 \\
\hline Total & 14 & 3 & 1 & 5 & 3 & 1 & 1 \\
\hline
\end{tabular}

Fuente: Base de Datos Scopus

El artículo que ha sido citado en 4 ocasiones fue Tourism, outdoor recreation and public use in natural parks. A proposal for the conservation of the environmental values and the local economic development [Turismo, actividades recreativas y uso público en los parques naturales. Propuesta para la conservación de los valores ambientales y el desarrollo productivo local]

Tabla 4. Artículos más citados según Scopus

\begin{tabular}{lc}
\hline \multicolumn{1}{c}{ Artículos } & Citas \\
\hline $\begin{array}{l}\text { Citizens' revolution and international integration } \\
\text { Influence of light interception on vegetative and productive development of peaches cv. Elegant Lady }\end{array}$ & 1 \\
[Influencia de la intercepción luminica en el desarrollo vegetativo y productivo de durazneros cv. & \\
elegant lady] & \\
$\begin{array}{l}\text { Tourism, outdoor recreation and public use in natural parks. A proposal for the conservation of the } \\
\text { environmental values and the local economic development [Turismo, actividades recreativas y uso } \\
\text { público en los parques naturales. Propuesta para la conservación de los valores ambientales y el } \\
\text { desarrollo productivo local] }\end{array}$ & 4 \\
$\begin{array}{l}\text { Untersuchungen über die Nutzleistung des Djallonkéschafes: Ein Beitag zur Kenntnis einer } \\
\text { westafrikanischen Schafrasse }\end{array}$ & 1 \\
\hline
\end{tabular}

Fuente: Base de Datos Scopus

El 28,6\% corresponde a la temática de agricultura y veterinaria (Gráfico 1).

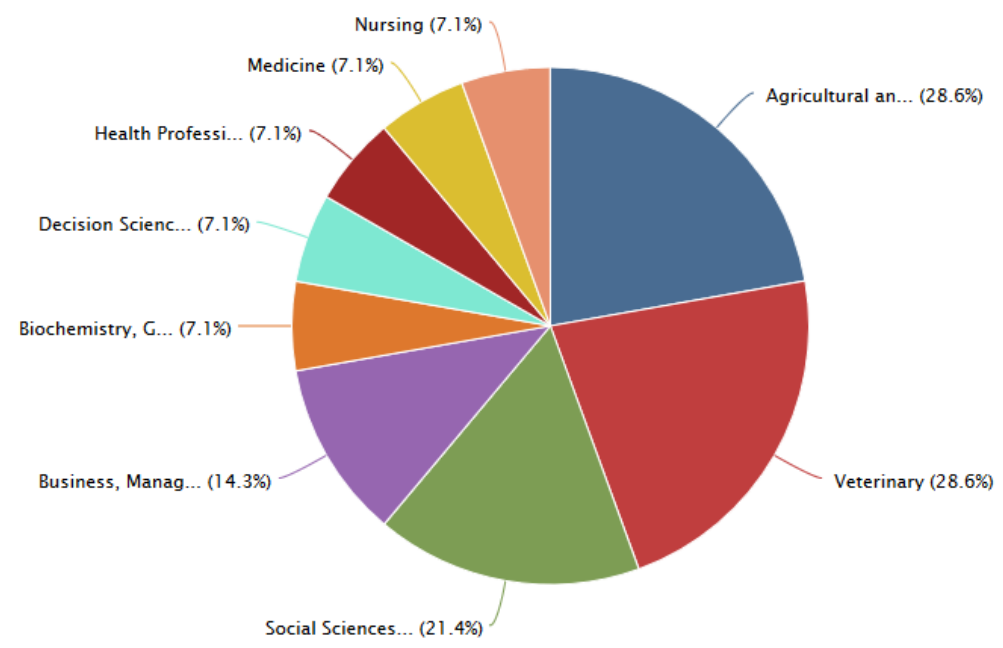

Gráfico 1. Documentos por áreas

Destacándose por número de citas e impacto la revista Cadernos de Saude Publica de Brasil, estando en el cuartil Q1 en temas de medicina a partir del 2007, y en temas de salud 
pública o salud ocupacional forma parte del cuartil Q2; siendo el 35\% de la participación de latinoamérica (Gráfico 2)

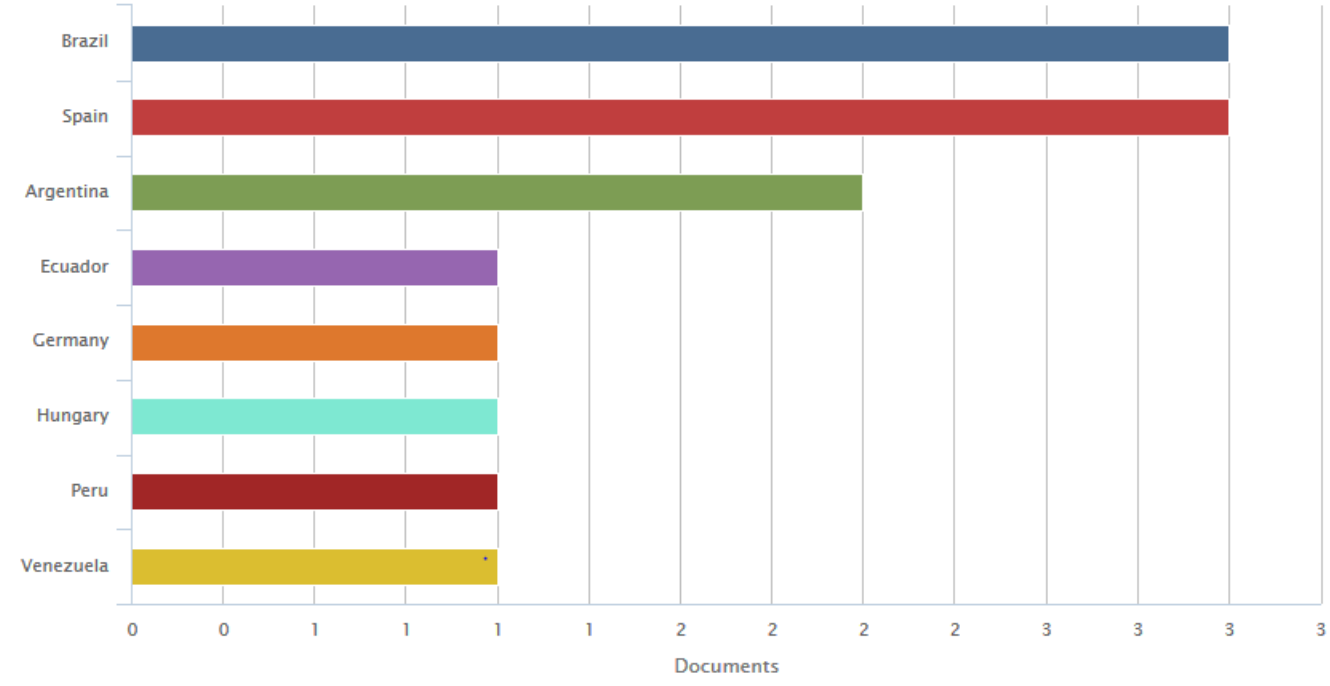

Gráfico 2. Documentos por países

\section{Resultados - "Desarrollo productivo" "Gremio de artesanos"}

La afiliación de University College Dublin, ha sido en 2 artículos; además comprende $44 \%$ de Estados Unidos (Gráfico 3) y el 55\% en el área de economía, econometría y finanzas (Gráfico 4).

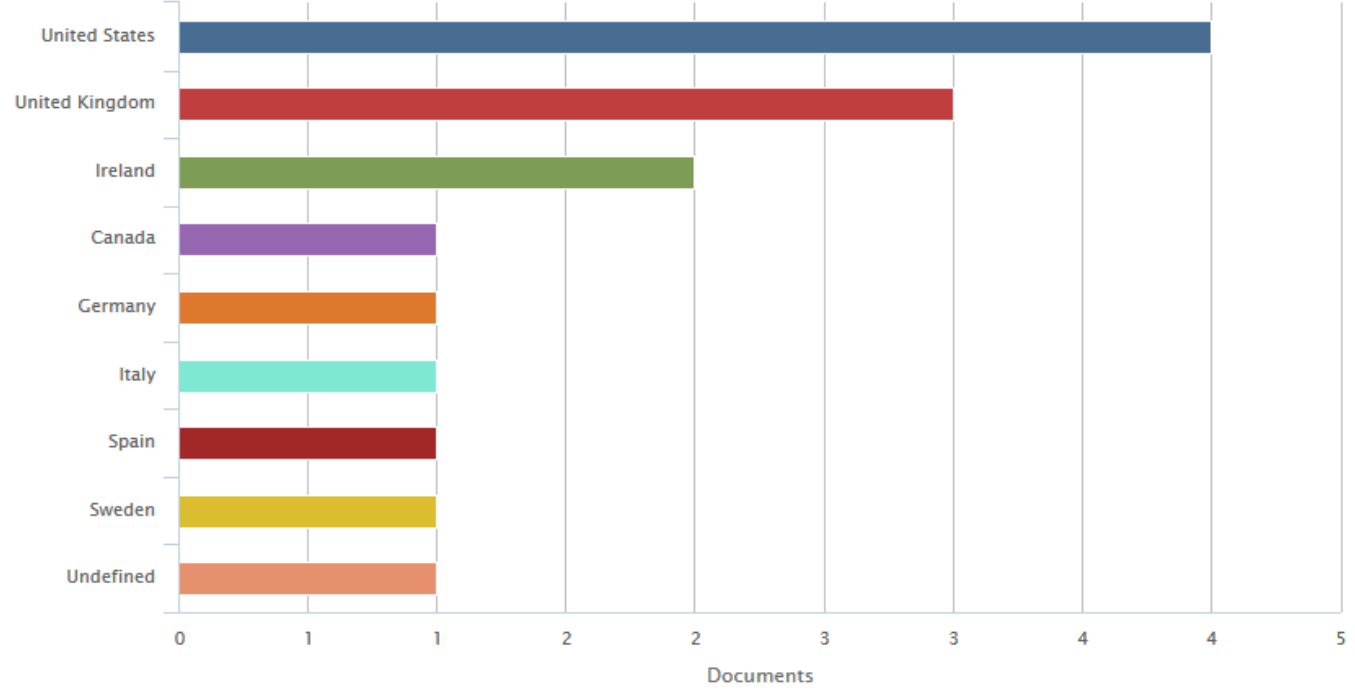

Gráfico 3. Documentos por países 


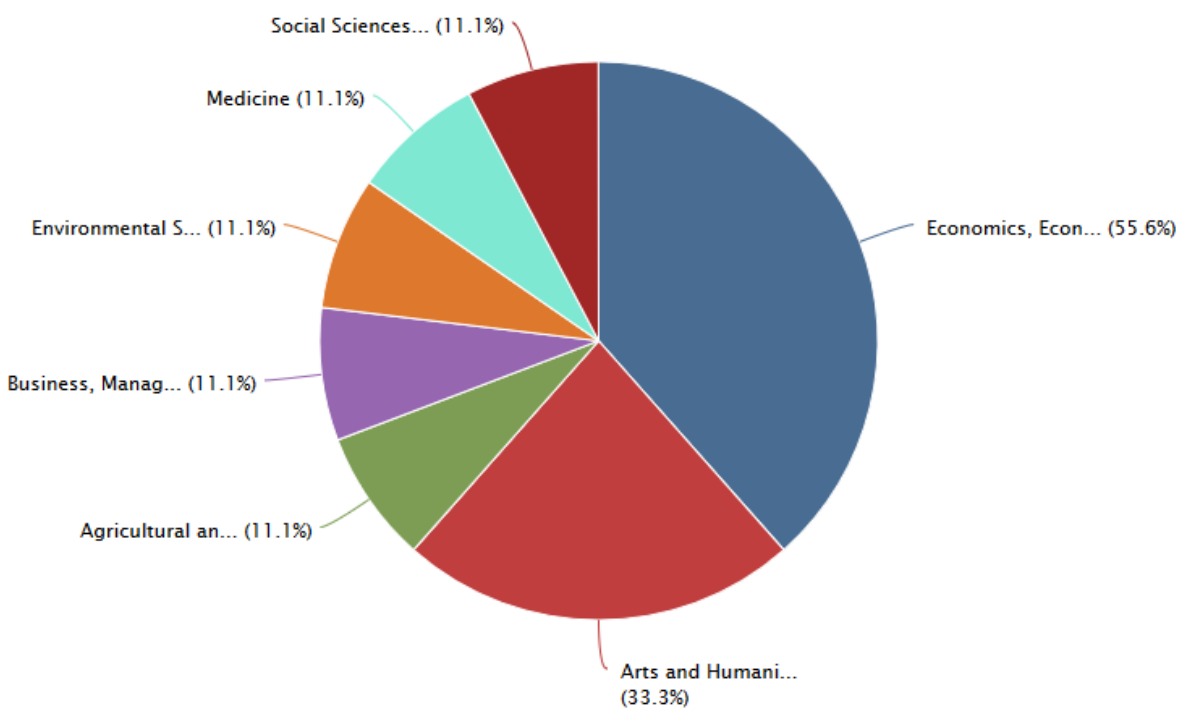

Gráfico 4. Documentos por áreas.

Como se observa en el Gráfico 5, se planteó modelado de objetos para identificación y sugerir aspectos tecnológicos de un software con la base del Lenguaje Unificado de Modelamiento (UML) que incluya sistematizar de búsqueda bibiométrica.

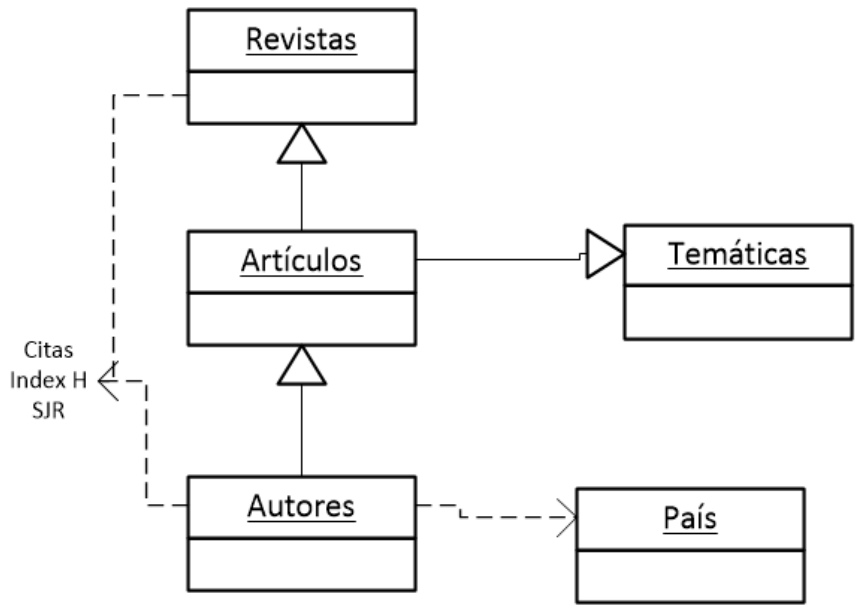

Gráfico 5. Análisis del estudio bibliométrico

De las 9 revistas el 77\% forman parte del cuartil Q1 según SJR, Scimago Journal Rank como factor de medir calidad de las publicaciones científicas con el recuento de citas en cada publicación, contabiliza el número de citas recibidas con el prestigio de las citas, además se presenta el CiteScore por el número de citaciones recibida y el índice $\mathrm{H}$ valorando la producción en función de las citas recibidas (Tabla 5). 
Tabla 5. Categoría de las revistas según Scopus y calificación en SJR

\begin{tabular}{|c|c|c|c|c|c|c|}
\hline Revistas & INDEX H & Categoría & Cuartil & SJR & $\begin{array}{l}\text { Cites per } \\
\text { doc }\end{array}$ & $\begin{array}{l}\text { Total } \\
\text { cites }\end{array}$ \\
\hline Annual Review of Economics & 29 & & Q1 & 5.72 & 2.27 & 228 \\
\hline Economic History Review & 37 & $\begin{array}{l}\text { Economía y } \\
\text { Econometría }\end{array}$ & Q1 & 1.21 & 1.23 & 162 \\
\hline Forest Ecology and & 140 & $\begin{array}{l}\text { Historia } \\
\text { Sivicultura }\end{array}$ & $\begin{array}{l}\text { Q1 } \\
\text { Q1 }\end{array}$ & 1.62 & 3.33 & 5949 \\
\hline & & $\begin{array}{l}\text { Administració } \\
\mathrm{n}, \quad \text { Política y } \\
\text { Leyes } \\
\text { Conservación }\end{array}$ & Q1 & & & \\
\hline $\begin{array}{l}\text { Handbook of Economic } \\
\text { Growth }\end{array}$ & 23 & & Q1 & 8.13 & 4.06 & 65 \\
\hline Historical Journal & 25 & & Q1 & 0.26 & 0.36 & 58 \\
\hline Journal of Management & 164 & $\begin{array}{l}\text { Finanzas } \\
\text { Estrategias de } \\
\text { administración }\end{array}$ & $\begin{array}{l}\mathrm{Q} 1 \\
\mathrm{Q} 1\end{array}$ & 5.83 & 6.09 & 1658 \\
\hline Journal of Public Health & 65 & & Q2 & 0.65 & 0.95 & 492 \\
\hline $\begin{array}{l}\text { Review of Economic } \\
\text { Dynamics }\end{array}$ & 50 & & Q1 & 3.04 & 1.3 & 219 \\
\hline $\begin{array}{l}\text { Studies in Global Social } \\
\text { History }\end{array}$ & 2 & & Q3 & 0.1 & 0 & 0 \\
\hline
\end{tabular}

Fuente: Base de Datos Scopus y SJR

\section{Conclusiones}

El trabajo de análisis bibliográfico efectuado en SCOPUS se ejecutó con interés de indagar artículos relacionados a las variables de "desarrollo productivo" y "gremios artesanales", analizándose 14 documentos con la consulta "desarrollo productivo" y 9 artículos con la búsqueda de "productive AND development AND craft AND guild" desarrollándose para este trabajo dos fases: 1 . Obtener información delimitad por variables, 2 . Evaluación y verificación de información para desarrollar un análisis básico de los resultados obtenidos, se ha concluir lo siguiente:

De las 14 publicaciones ha existido la participación de un artículo en el 2017 de 10 autores con el título Replacing soybean for winter peas in heavy-pig diets: Productive impact of the level of protease inhibitors [Sustitución de soja por guisante de invierno en dietas de cerdos pesados: Impacto productivo del nivel de inhibidores de proteasas] en la revista ITEA Informacion Tecnica Economica Agraria, revista que forma parte del cuartil Q3, según SJR con valor de 0,26 al 2016, con un total de citas de 41; en comparación con las revistas publicadas en el 2016: Cadernos de Saude Publica y la revista Latin American Perspectives según SJR se encuentran en el cuartil Q1, y la revista Revista de Administracao Publica, se encuentra en el cuartil Q3 con la participación de 3 autores.

El artículo Citizens' revolution and international integration, además de encontrarse en un revista del cuartil Q1, aunque se encuentra el artículo Tourism, outdoor recreation and public 
use in natural parks. A proposal for the conservation of the environmental values and the local economic development [Turismo, actividades recreativas y uso público en los parques naturales. Propuesta para la conservación de los valores ambientales y el desarrollo productivo local] con 4 citas pero forma parte del Boletin de la Asociacion de Geografos Espanoles, catalogado en el cuartil Q4. Por el número de citas e impacto la revista Cadernos de Saude Publica de Brasil existe el $35 \%$ de la participación de Latinoamérica.

Scimago Journal Rank mide la calidad de publicaciones científicas considera el número de citas en cada publicación con el prestigio de las citas, el CiteScore el número de citaciones recibida y el índice $\mathrm{H}$ valora la producción en función de las citas recibidas, destacándose el artículo Precocious albion: A new interpretation of the British Industrial revolution, publicada en Annual Review of Economics en el 2014 por Kelly, Mokyr, and Gráda, resalta la importancia del capital humano y las causas de la mayor calidad del trabajo están asociadas con un alto nivel de nutrición, mejores instituciones, y un alto nivel de aprendizaje.

\section{Bibliografía}

Bodas, R., López-Campos, O., \& Mantecón, A. R. (2009). Parda de Montaña replacement heifers: Nutrition and productive performance [Recría de terneras parda de montaña: Alimentación y desarrollo productivo]. Archivos de Zootecnia, 58(222), 309-312. Retrieved from https://www.scopus.com/inward/record.uri?eid=2-s2.067549123553\&partnerID=40\&md5=02a5050736cd8160fc8aa0fb9d017ca3

Brudvig, L. A., Grman, E., Habeck, C. W., Orrock, J. L., \& Ledvina, J. A. (2013). Strong legacy of agricultural land use on soils and understory plant communities in longleaf pine woodlands. Forest Ecology and Management, 310, 944-955. https://doi.org/10.1016/j.foreco.2013.09.053

Bruno Buqueras, C. (1981). Logopedia, integración y deficientes auditivos. "Revista de Logopedia, Foniatria Y Audiologia," 1(2), 68-77. https://doi.org/10.1016/S02144603(81)75195-7

Burt, E L, and J Atkinson. "The Relationship between Quilting and Wellbeing." Journal of Public Health 34.1 (2012): 54-59. Web.

Clavé, S. A., Boqué, J. B., \& Salvat, J. (2008). Tourism, outdoor recreation and public use in natural parks. A proposal for the conservation of the environmental values and the local economic development [Turismo, actividades recreativas y uso público en los parques naturales. Propuesta para la conservación de los valores ambientales y el desarrollo productivo local]. Boletin de La Asociacion de Geografos Espanoles, (48), 5-396. Retrieved from https://www.scopus.com/inward/record.uri?eid=2-s2.058149329114\&partnerID=40\&md5=3223bdde55fb2fc623197023793adc 16

Costen, M., Slavin, P., Paul, H., Walsh, P., Crook, T., Velkar, A., \& Godden, C. (2015). Review of periodical literature published in 2013. Economic History Review, 68(1), 286-353. https://doi.org/10.1111/ehr.12107 
Desmet, K., \& Parente, S. L. (2014). Resistance to technology adoption: The rise and decline of guilds. Review of Economic Dynamics, 17(3), 437-458. https://doi.org/10.1016/j.red.2013.09.005

Dussi, M. C., Giardina, G., Giménez, G., Reeb, P., Zon, K., \& Nyeki, J. (2009). Influence of light interception on vegetative and productive development of peaches cv. Elegant Lady [Influencia de la intercepción lumínica en el desarrollo vegetativo y productivo de durazneros cv. elegant lady]. Revista de La Facultad de Ciencias Agrarias, 41(1), 155-164. Retrieved from https://www.scopus.com/inward/record.uri?eid=2-s2.075949106159\&partnerID=40\&md5=ca8cb3f73777ece0939502797ef47c7b

Filius, P., Weniger, J. H., \& Teuscher, T. (1985). Untersuchungen über die Nutzleistung des Djallonkéschafes: Ein Beitag zur Kenntnis einer westafrikanischen Schafrasse. Journal of Animal Breeding and Genetics, 102(1-5), 371-384. https://doi.org/10.1111/j.14390388.1985.tb00706.x

Gedajlovic, E., Carney, M., Chrisman, J. J., \& Kellermanns, F. W. (2012). The Adolescence of Family Firm Research: Taking Stock and Planning for the Future. Journal of Management, 38(4), 1010-1037. https://doi.org/10.1177/0149206311429990

Gil, L., White, A., \& Caceres, E. (1990). Financing research in Chile in biotechnology and other areas related to the productive sector [Financiamiento de la investigación en Chile en biotecnología y en otras áreas relacionadas con el sector productivo.]. Archivos de Biologia Y Medicina Experimentales, 23(2), 77-87. Retrieved from https://www.scopus.com/inward/record.uri?eid=2-s2.00025495594\&partnerID=40\&md5=b46a0df376f760e5dab831ce93f59512

Gómez-Izquierdo, E., De Mercado, E., Gómez-Fernández, J., Tomás, C., Guillamón, E., Varela, A., ... Latorre, M. A. (2017). Replacing soybean for winter peas in heavy-pig diets: Productive impact of the level of protease inhibitors [Sustitución de soja por guisante de invierno en dietas de cerdos pesados: Impacto productivo del nivel de inhibidores de proteasas]. ITEA Informacion Tecnica Economica Agraria, 113(2), 138-157. https://doi.org/10.12706/itea.2017.009

Jacometti, M., Castro, M., Gonçalves, S. A., \& Costa, M. C. (2016). An analysis of the effectiveness of public policies relating to local productive arrangements (LPA'S) on local development from the perspective of institutional theory [Análise de efetividade das políticas públicas de arranjo produtivo local para o desenvolvimento local a partir da teoria institucional] [Análisis de la efectividad de las políticas públicas de arreglo productivo local para el desarrollo local desde la teoría institucional]. Revista de Administracao Publica, 50(3), 425-454. https://doi.org/10.1590/0034-7612142712

Kelly, M., Mokyr, J., \& Gráda, C. Ó. (2014). Precocious albion: A new interpretation of the British Industrial revolution. Annual Review of Economics, 6, 363-389. https://doi.org/10.1146/annurev-economics-080213-041042 
Lis, C., \& Soly, H. (2012). Worthy efforts: Attitudes to work and workers in pre-industrial Europe. Studies in Global Social History, 10, 1-678. Retrieved from https://www.scopus.com/inward/record.uri?eid=2-s2.084964757043\&partnerID=40\&md5=e0531c84092dc31a23493f2fa204b832

Moré, J. D., Lima, F. G. F., \& de Almeida, L. N. (2010). Model management development plan local production: The case of Nova Friburgo, Brazil [Modelo de gestión de un plan de desarrollo productivo local: El caso de Nova Friburgo, Brasil]. Journal of Technology Management and Innovation, 5(3), 192-205. Retrieved from https://www.scopus.com/inward/record.uri?eid=2-s2.078549236563\&partnerID=40\&md5=995a7ab52ce451d940643818e2b0e675

Ogilvie, S., \& Carus, A. W. (2014). Institutions and Economic Growth in Historical Perspective. Handbook of Economic Growth, 2, 403-513. https://doi.org/10.1016/B978-0-444-535382.00008-3

Oleas-Montalvo, J. (2016). Citizens' revolution and international integration. Latin American Perspectives, 43(1), 124-142. https://doi.org/10.1177/0094582X15575693

Osta, K. M. (2007). Franchises: Enterprise networks and relations for the productive development [Franquicias: Redes y relaciones empresariales para el desarrollo productivo]. Espacios, 28(2), 19-21. Retrieved from https://www.scopus.com/inward/record.uri?eid=2-s2.084874572500\&partnerID=40\&md5=b3b20601d64da6ad179b6b93b9db7ced

P. Willging, G. J. Astudillo y S. G. Bast, «SEDICI de la Universidad Nacional de la Plata,» de El software de animación como una estrategia innovadora para el aprendizaje de lenguajes de programación, Buenos Aires, 2012.

Pihl, C. (2015). Gender, Labour, And State Formation In Sixteenth-Century Sweden. Historical Journal, 58(3), 685-710. https://doi.org/10.1017/S0018246X15000023

Salvador, E., \& Guevara, V. (2013). Development and validation of a model to predict the optimum requirement of essential amino acids and performance in commercial laying hens [Desarrollo y validación de un modelo de predicción del requerimiento óptimo de aminoácidos esenciales y del comportamiento productivo en ponedoras comerciales]. Revista de Investigaciones Veterinarias Del Peru, 24(3), 264-276. Retrieved from https://www.scopus.com/inward/record.uri?eid=2-s2.0-

84883564607\&partnerID=40\&md5=538594f3d52bc6b5097555d57552b7e1

Saubidet, A., Pérsico, M. M., Lucero, M., \& Imeroni, J. (2012). Productive, didactic module for the development of outdoor learning practices in the career of Technician in Aquaculture and Fisheries Processing [Módulo didáctico-productivo para el desarrollo de prácticas de aprendizaje extráulicas, en la carrera Tecnicatura en Acuicultura y Procesamiento Pesquero]. Revista Electronica de Veterinaria, 13(12). Retrieved from https://www.scopus.com/inward/record.uri?eid=2-s2.0-

84874634130\&partnerID=40\&md5=b4b797ed75f4feba2264f7f4fb9a1718 
Viana, A. L. D., da Silva, H. P., Ibañez, N., \& Iozzi, F. L. (2016). Development policy for the Brazilian health industry and qualification of national public laboratories [A política de desenvolvimento produtivo da saúde e a capacitação dos laboratórios públicos nacionais] [La política de desarrollo productivo en salud en Brasil y la cualificación de los laboratorios públicos nacionales]. Cadernos de Saude Publica, 32. https://doi.org/10.1590/0102$311 \mathrm{X} 00188814$ 